\title{
Scalp psoriasis associated with Malassezia species
}

\author{
Bogomolova T.S., Kornisheva V.G., Bogdanova T.V., Gulordava M.G., Smolina O.A., Alexeev A.Y. \\ Kashkin Research Institute of Medical Mycology of North-Western State Medical University named after I.I. Mechnikov, \\ St. Petersburg, Russia
}

\section{Objectives}

Scalp involvement in patients with psoriasis is one of the most frequent manifestations of the disease. The role of Malassezia species in psoriasis is still undetermined. These lipophilic yeasts may be a trigger factor. The aim of the study was to investigate the presence of Malassezia species colonization in scalp lesions of patients with psoriasis and to determine its dependence on the size of lesions.

\section{Methods}

A prospective study of 144 patients with scalp psoriasis observed at the Department of Dermatology in Mycological Clinic during the period $2013-2017$ (Fig. 1). 46 men and 98 women aged 18 - 74 years were examined. The patients were divided into two groups: (I) those in whom psoriatic area of the scalp was $10 \%-29 \%$ of the whole scalp surface (34), and (II) in whom psoriatic area of the scalp was $30 \%-89 \%$ (110). All patients were evaluated for the presence of Malassezia species on the scalp lesions. Scrapings from the surface of the scalp lesions and strips of cellophane tape containing clinical specimens from patients were investigated by direct fluorescent microscopy with Calcofluor White (Fig. 2) and by culture on the modified Leeming and Notman agar ( $\mathrm{mLNA}$ ) using contact plates for surface microbial sampling [Chander J., 2017].

\section{Results}

Malassezia spp. were detected in $83 \%$ (119) of patients. Both tests (direct microscopy and culture) were positive in 61\% (88) of patients. Cultures were positive in $82 \%(98)$ of cases (Fig. 3). Monoculture Malassezia spp. was identified in 85 patients (71\%), association with Candida sp. in 5 patients, association with Rhodotorula sp. - in 8 patients. Malassezia species were found in $12 \%$ of the I group patients and in $76 \%$ of the II group patients. The prevalence of Malassezia species according to the severity of the scalp involvement differed significantly $(p<0,001)$.

\section{Conclusion}

Thus, frequency of Malassezia species presence on the scalp increases with the enlargement of the psoriatic area. At follow-up we recommend laboratory investigations for the presence of Malassezia species in lesions of patients with scalp psoriasis twice a year.
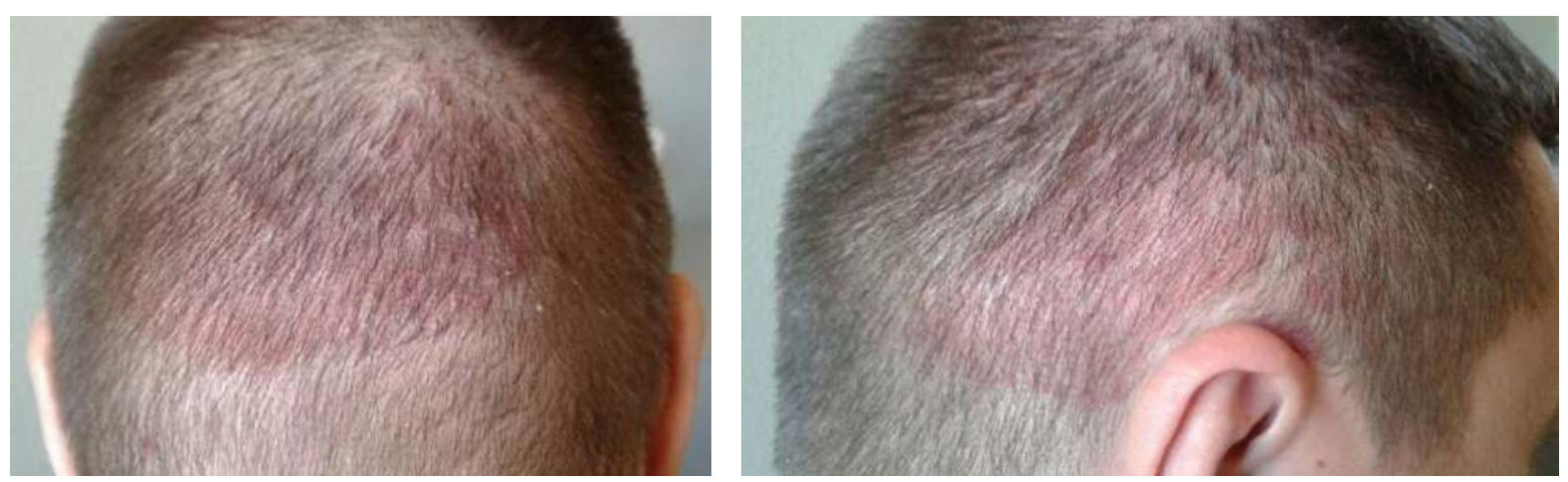

Fig. 1. Patient with scalp psoriasis.

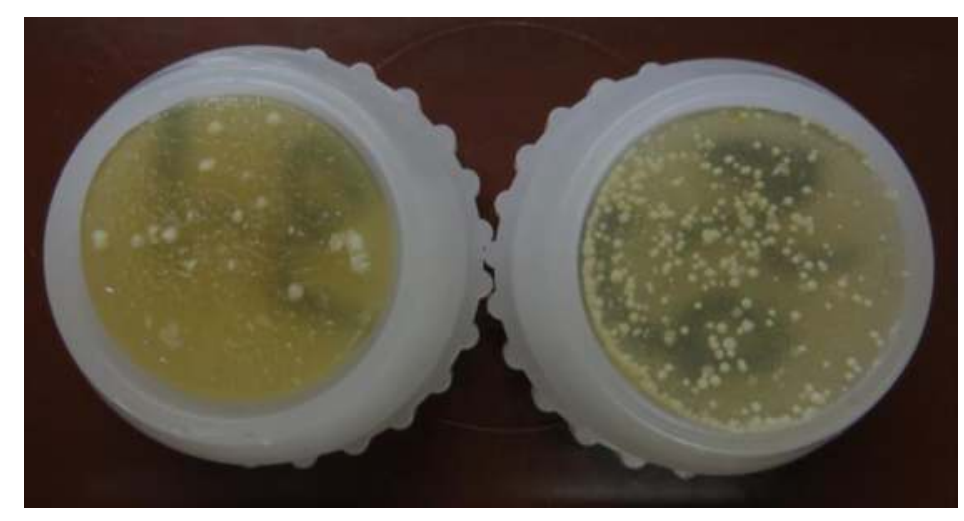

Fig. 3. Contact plates with growth of Malassezia sp. colonies on $m L N A$ : $a$ - healthy skin area on the scalp; $b$ - psoriatic area on the scalp of the same patient.
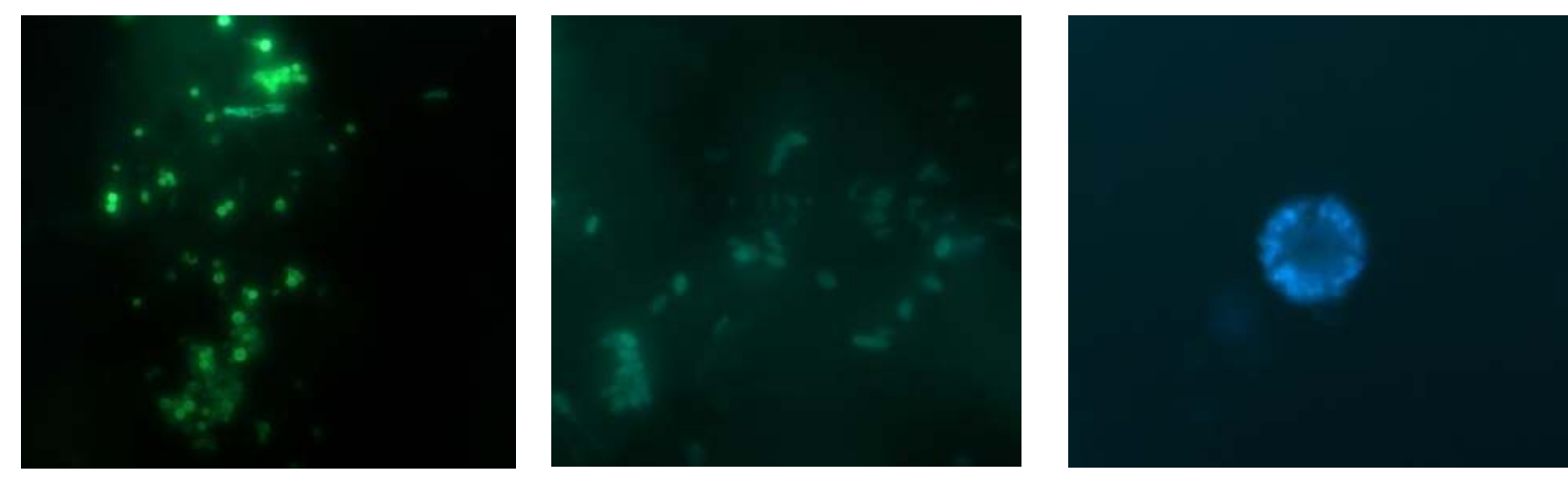

Fig. 2. Direct fluorescent microscopy of skin scrapings from patients with scalp psoriasis: $a$ - round yeast cells; $b$ - elongated yeast cells; c - yeast cells inside sebum lipid droplet. 